\title{
Note
}

\section{Clarification of some mathematical misunderstandings about Savage's foundations of statistics, 1954}

\author{
Peter Wakker \\ University of Nijmegen (NICI), Nijmegen $6500 \mathrm{HE}$, Netherlands \\ Communicated by $\mathrm{H}$. Gottinger \\ Received 3 September 1991 \\ Revised 2 April 1992
}

\begin{abstract}
This note discusses some mathematical misunderstandings about Savage (1954). It is shown that in his model the probability measure cannot be countably additive, that the set of events must be a $\sigma$-algebra and not just an algebra, that Savage did not characterize all atomless finitely additive probability measures, and that the state space in his model, while infinite, does not have to be uncountable.
\end{abstract}

Key words: Probability measure; $\sigma$-algebra; finite additivity.

In this note we discuss some mathematical misunderstandings about Savage (1954). We also clarify some misunderstandings that might arise in relation to the recent Lehrer (1991). That paper gives the most general result on qualitative probability theory, presently available in the literature, by extending earlier results of Chateauneuf (1985). The results of Section 6 there, however, should not be related to Savage's set-up, as examples will demonstrate. Those results hold true, and were meant ${ }^{1}$ only for countably additive probability measures with $\sigma$-algebras as domain. This note shows that they do not apply to general finitely additive probability measures, as in Savage's set-up. The observations of this note will not be surprising to the reader acquainted with the intricacies of finite additivity as opposed to countable additivity. Nevertheless, given the importance of Savage's work, and the frequency of the misunderstandings, the work seems worthwhile. Usually students are taught the properties of countably additive measures, not of finitely additive measures. As

Correspondence to: P. Wakker, University of Nijmegen (NICI), Psychological Laboratory, P.O. Box 9104, 6500 HE Nijmegen, Netherlands.

\footnotetext{
1 This was pointed out in Lehrer (1991, personal communication).
} 
a consequence, often scientists erroneously apply results, only valid for countably additive measures, to finitely additive measures. An extensive work dealing with finite additivity is Bhaskara Rao and Bhaskara Rao (1983).

Savage (1954) assumes that $\mathscr{A}$, the collection of events under consideration, contains all subsets of $S$, where $S$ denotes the state space. Working with the collection of all subsets is not customary in probability theory. One problem is that it is an open question whether there exists an atomless countably additive probability measure on a collection of all subsets of a set. In particular this means that it is not known if it is at all possible to have the probability measure countably additive in Savage's set-up! That possibility depends on the axioms from set-theory that one accepts:

Observation 1. In Savage's (1954) model, where all subsets of $S$ are events, the probability measure cannot be countably additive if the continuum hypothesis is added to the set-theoretic axioms.

The continuum hypothesis entails that there is no cardinality between that of the natural numbers and the reals. See Jech (1978, Corollary 1 of Lemma 27.9). If one does not accept the continuum hypothesis and assumes existence of a countably additive probability measure in Savage's set-up, then there is no constructive way to obtain that probability measure. That is, if they exist, we will never meet them. Savage avoided the assumption that $\mathscr{A}$ is a general $\sigma$-algebra only for simpliticy of presentation, to make his work accessible to readers not acquainted with measuretheory. This is suggested in Savage (1954, p. 40, 1.25/26 'except for expository complications...'). All of Savage's results and derivations remain valid if $A$ assumed to be a general $\sigma$-algebra; see Savage (1954, p. 40,1.24/25 there is no technical obstacle to...'). According to Savage (1954, p. 43, first paragraph) it is crucial though that be a $\sigma$-algebra, not just an algebra. In spite of this explicit statement, many misunderstandings have arisen in relation to this point. Wakker (1981) pointed out several of these.

Observation 2. Savage's analysis remains valid if 1 is taken to be a $\sigma$-algebra. This is not the case if $\mathscr{A}$ is an algebra.

Section 6 in Lehrer (1991) may lead to the misunderstanding that Savage would have characterized all relations on any algebra which can be represented by an atomless probability measure. This is not the case, as will be demonstrated below. Lehrer intended his results only for countably additive probability measures; Savage (1954) deals with finitely additive probability measures. Throughout what follows, $\lambda$ denotes the Lebesgue measure on $[0,1]$. An atom is an event with positive measure, such that any subevent has either that same measure, or measure 0 .

Example 1. Let $S=(0,1]$. Let $P^{\prime}=\lambda / 3+2 w / 3$, where $w$ is the atomic measure assigning measure 1 to $\{1 / 2\} . P^{\prime}$ is defined on the Borel subsets, where $\{1 / 2\}$ is an 
atom. For $\mathscr{A}$, however, we take the algebra of all finite unions of left-open rightclosed intervals. $P$ is the restriction of $P^{\prime}$ to $\mathscr{A} . P$ is atomless. It does not satisfy the axioms of Savage, in particular fineness does not hold. Note that $Q=\lambda / 4+3 w / 4$ represents the same ordering on $\mathscr{A}$ as $P$.

The representation $P$ in the above example was not uniquely determined. So Theorem 5 and Corollary 2 in Lehrer (1991) cannot be extended to finitely additive probability measures on algebras. In the following example $A$ will contain all subsets. It will thus be as in Savage (1954), and obviously is a $\sigma$-algebra.

Example 2. Let $S=(0,1]$, and $\mathscr{A}$ be the collection of all subsets of $S$. With slight abuse of notation, we denote by $\lambda$ an extension, finitely additive, of the Lebesgue measurc to $\mathscr{A} . \lambda$ is atomless since $S$ can be split up in arbitrarily fine 'uniform' partitions, i.e. partitions containing equally-probable events. Let $w$ be a finitely additive measure on all subsets of $(0,1]$, still to be defined. It will only assign measures 0 or 1 . We take $w$ to be absolutely continuous with respect to $\lambda$, i.e. any $\lambda$-null set has $w$-measure 0. Furthermore, we set $w((0, \varepsilon])=1$ and $w((\varepsilon, 1])=0$ for all $\varepsilon>0$. Obviously this already suffices to assure that $w$ is not countably additive, even if its domain would only contain Borel sets. This $w$ can be extended to all subsets of $S$, in many ways, by means of the Hahn-Banach extension theorem, or the choice axiom, such that only measures 0 and 1 are assigned. (The sets with $w$-measure 1 constitute an 'ultrafilter' containing, among others, all sets with $\lambda$-measure 1.) Finally $P:=\lambda / 3+2 w / 3$. This $P$ is atomless, since any set with positive $P$-measure must have positive $\lambda$-measure, and so must have a subset with strictly smaller, but positive, $P$ measure. Savage's axioms are not satisfied, in particular fineness is violated. $Q:=$ $\lambda / 4+3 w / 4$ represents the same ordering on $P$ as $P$.

The above example shows that, also if the algebra of events contains all subsets, then Theorem 5 and Corollary 2 of Lehrer (1991, Section 4) still hold true only for countably additive probability measures. Furthermore, it shows that 'convexrangedness' of the probability measure (for each event $A$, and $0 \leq \mu \leq P(A)$, there exists an event $B \subset A$ with $P(B)=\mu$ ), while equivalent to atomlessness for countably additive probability measures, for finitely additive probability measures is more restrictive than atomlessness.

Observation 3. Savage did not characterize all atomless finitely additive probability measures.

Savage (1954) did not give conditions that on general $\sigma$-algebras would imply countable additivity of the probability measure. For qualitative probability theory such a condition was given in Villegas (1964). He characterized all atomless count ably additive probability measures on $\sigma$-algebras. Arrow (1965) used this to give an 
elegant presentation of Savage's (1954) result on general $\sigma$-algebras which does incorporate countable additivity.

Let us take the opportunity to discuss one more misunderstanding. The following example proves Observation 4.

Example 3. Let $S=(0,1] \cap \mathbb{Q}, \mathscr{A}$ contains all subsets of $S$. Let $P$ assign the length of any interval to its intersection with $S . P$ can be extended to $\mathscr{A}$. $P$ satisfies all of Savage's conditions, and obviously is not countably additive. Even while for each $B \subset S$ and $0 \leq \mu \leq P(B)$ there is $C \in \mathscr{A}$ with $P(C)=\mu, S$ is countable. If $[0,1]$ is the set of consequences and utility is identity, then expected utility maximization satisfies all of Savage's postulates for preferences.

Observation 4. Savage's axioms do not imply that the state space be uncountable.

Finally we mention that in Wakker (1991) it is shown that Savage's axioms usually necessitate a violation of stochastic dominance in the traditional sense, and that therefore for finitely additive probability measures an alternative definition of stochastic dominance is warranted.

\section{Acknowledgement}

The research has been made possible by a fellowship of the Royal Netherlands Academy of Arts and Sciences.

\section{References}

Arrow, K.J., Aspects of the Theory of Risk-Bearing (Academic Bookstore, Helsinki, 1965). Elaborated as Arrow, K.J., Essays in the Theory of Risk-Bearing (North-Holland, Amsterdam, 1971).

Bhaskara Rao, K.P.S. and M. Bhaskara Rao, Theory of Charges (Academic Press, London, 1983).

Chateauneuf, A., On the existence of a probability measure compatible with a total preorder on a Boolean algebra, J. Math. Econom. 14 (1985) 43-52.

Jech, T., Set Theory (Academic Press, New York, 1978).

Lehrer, E., On a representation of a relation by a measure, J. Math. Econom. 20 (1991) 107-118.

Savage, L.J., The Foundations of Statistics (Wiley, New York, 1954) (Second edition: Dover, New York, 1972).

Villegas, C., On quantitative probability $\sigma$-algebras, Ann. Math. Statistics 35 (1964) 1787-1796.

Wakker, P.P., Agreeing probability measures for comparative probability structures, Ann. Statistics 9 (1981) 658-662.

Wakker, P.P., Savage's axioms usually imply violation of strict stochastic dominance, University of Nijmegen, NICI, Nijmegen, Netherlands, 1991. 